\title{
Acute Cauda Equina Syndrome after Percutaneous Transforaminal Endoscopic Discectomy
}

\author{
Mohammad I. Badra ${ }^{1}$, Mohammad H. Jawad Rahal ${ }^{1,2}$, Elie Najjar ${ }^{3}$, Nizar Y. Natout ${ }^{1}$ \\ ${ }^{1}$ Department of Orthopedic Surgery and Traumatology, Clemenceau Medical Center Affiliated With Johns Hopkins International, \\ Clemenceau, Beirut; ${ }^{2}$ Department of Orthopedic Surgery and Traumatology, Lebanese University, Faculty of Medical sciences, Beirut; \\ ${ }^{3}$ Department of Orthopedic Surgery and Traumatology, Lebanese American University, Faculty of Medicine, Beirut, Lebanon
}

Corresponding Author:

Mohammad Ibrahim Badra, MD

Department of Orthopedics Surgery, Clemenceau Medical Center Affiliated With Johns Hopkins International, Beirut 11-2555, Lebanon

Tel: +961-3-020322

Fax: +961-1-364464

E-mail: mbadra13@gmail.com

Received: July 14, 2018

Revised: September 25, 2018

Accepted: October 28, 2018

\begin{abstract}
Percutaneous Endoscopic Lumbar discectomy (PELD) has proved to be a good alternative to open micro-discectomy for treatment of lumbar disc herniation. This technique has been shown to be associated with several peri-operative complications and morbidities. These include postoperative dysesthesia secondary to nerve root injury, dural tears, hematoma, infection and recurrence of disc herniation. In this report, we are presenting a rare case of $L 4-5$ disc herniation that was treated surgically with PELD and was complicated by early recurrence causing acute cauda equina syndrome. Our aim is to highlight on this rare complication that should be taken into consideration by minimally invasive spine surgeons utilizing PELD in their practice.
\end{abstract}

Key Words: Percutaneous Endoscopic Lumbar Discectomy, Complications, Cauda equina syndrome

\section{INTRODUCTION}

Percutaneous Endoscopic Lumbar discectomy (PELD) is a rapidly evolving surgical technique for the treatment of disc herniation in the lumbar spine. As with any surgical technique, this procedure is associated with several complications such as infection, nerve injury, dural tears, bleeding and recurrence of the disc herniation. The rate of recurrence after PELD has been reported to be $0-7.4 \% \%^{8,16,18)}$. Although the rate of recurrence has been shown to be comparable to conventional discecto$m y^{5,111}$, many surgeons are concerned about early recurrence after percutaneous endoscopic discectomy. We present here a case of percutaneous endoscopic lumbar discectomy at L4-5 using a transforaminal approach under General anesthesia that was complicated by early recurrence causing acute cauda equina syndrome.

\section{CASE REPORT}

A 25 years old female patient, relatively obese (BMl 30), presented with a history of low back pain associated with right leg radicular pain of 2 months duration. Physical exam showed normal motor power (5/5) of all muscle groups in both lower extremities with normal sensation and normal deep tendon reflexes. She had no bowel or bladder related symptoms. Plain radiographs of the lumbar spine showed mild degenerative disc disease, mainly at L4-5 level.

Patient was started on medical treatment that included nonsteroidal Anti-inflammatory drugs (NSAID), muscle relaxants and Pregabalin. Follow up examination after 6 weeks showed minimal response to the medical treatment with persistence of the right leg pain and numbness. MRI ordered revealed degenerative disc disease at L4-5 level (pfirmann class IV) with a large right paracentral disc herniation compromising the spinal canal and the right lateral recess and compressing the right L5 nerve root (Fig. 1); Dynamic $X$-rays of lumbar spine with flexion and extension views showed stable lumbar vertebrae without evidence of spondylolisthesis or instability (Fig. 2). After discussing the surgical options with the patient, it was decided to proceed with Percutaneous Transforaminal Endoscopic Discectomy (PTED) at L4-L5 under General anesthesia. The procedure was done under fluoroscopy guidance in the usual manner with complete excision of the herniated disc fragments. At the end of the procedure, the nerve root was noted to be free floating without any residual compression (Fig. 3). Histopathological evaluation revealed fragments of degenerated fibrocartilage with attached hyaline cartilage and cartilage endplate.

Immediate post-op evaluation of the patient showed complete resolution of the symptoms with disappearance of the right leg pain and numbness and the patient was discharged on the same day. 


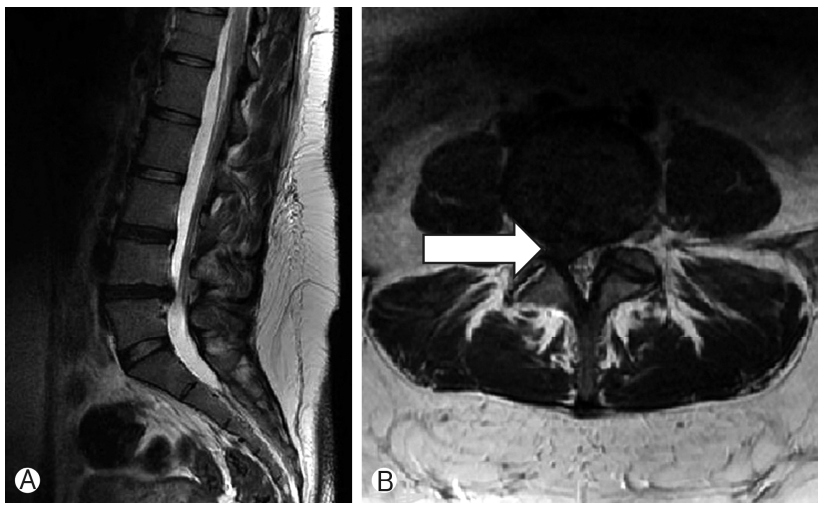

Fig. 1. Pre-op sagittal (A) and axial (B) T2W MRI pictures large right paracentral big annular tessar (arrow) L4-L5 disc herniation compressing the right $L 5$ nerve root and the thecal sac.
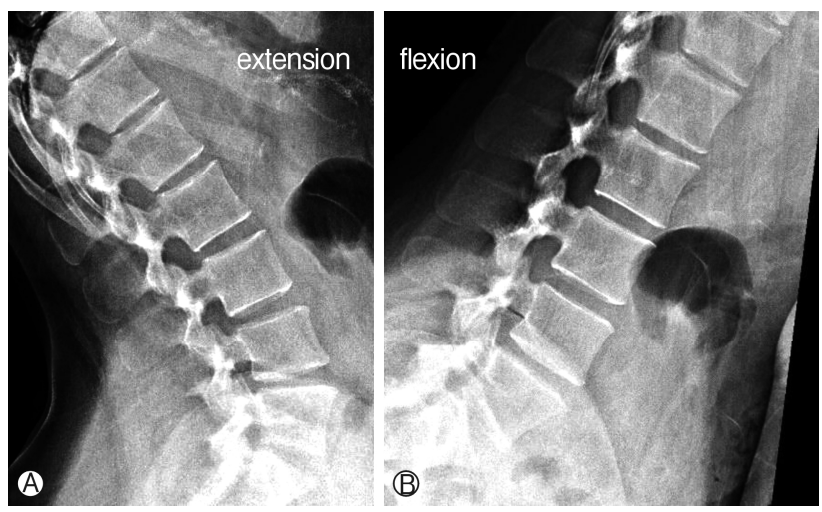

Fig. 2. Pre-op sagittal Dynamic X-rays of lumbar spine with extension (A) and flexion (B) views showed stable lumbar vertebrae without evidence of spondylolisthesis or instability.
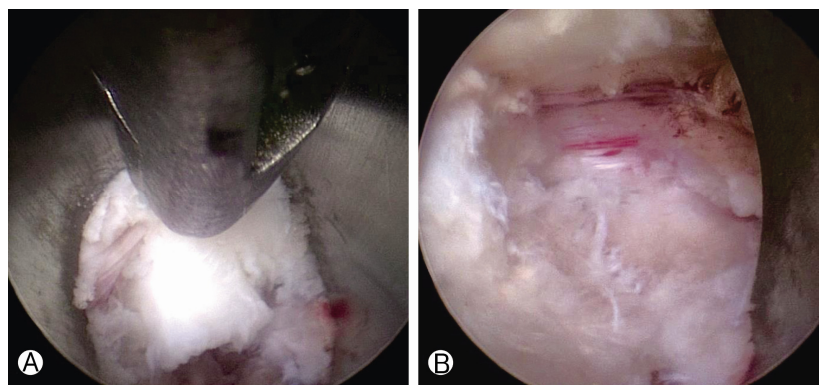

Fig. 3. Intraoperative endoscopic view of PETLD technique. (A) Extraction of Massive disc compressing thecal sac and Right L5 nerve root dorsally. (B) Free L5 nerve root observed after removal of disc.

On the $3^{\text {rd }}$ post-operative day, the patient started to have bilateral lower extremities numbness and weakness with decrease in her ability to ambulate at home. She denied any history of trauma or strenuous activities. Examination in the clinic showed bilateral foot drop, difficulty in ambulation without assistance, absent Achilles tendon reflexes bilaterally, saddle anesthesia
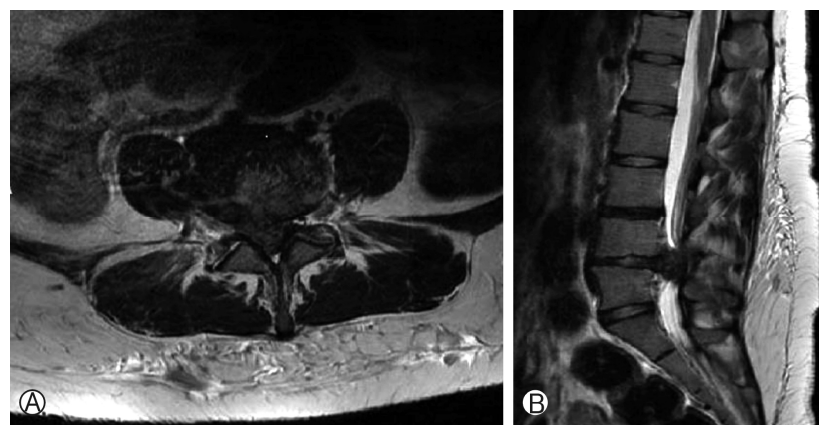

Fig. 4. Post op axial (A) and sagittal (B) T2W MRI pictures at L4-5 level showing recurrent large L4-L5 Disc Herniation obliterating the spinal canal and compressing Severely the Dural sac and neural elements.

and urinary incontinence suggestive of acute Cauda Equine Syndrome. MRI done on urgent basis showed recurrent large L4-L5 disc herniation obliterating the spinal canal and compressing severely the dural sac and neural elements (Fig. 4).

\section{Surgical Techniques}

The patient was transferred immediately to the operating room where surgical open laminectomy and decompression of the neural elements was done with complete discectomy at L4-L5 level.

Histopathological examination revealed again fragments of degenerated fibrocartilage with attached hyaline cartilage and cartilage endplate.

The patient showed daily improvement in her clinical status with decrease in the saddle anesthesia and numbness and regained motor power in her lower extremities. On the $3^{\text {rd }}$ postoperative day, the patient was discharged home on Pregabalin, anti-inflammatory medications and pain killers with physiotherapy of the lower extremities on daily basis.

Follow Up at 2weeks, 1month and 3 months showed continuous improvement in her lower extremities motor power with ambulatory function restored without assistance.

\section{DISCUSSION}

Percutaneous Endoscopic Lumbar Discectomy (PELD) is a new minimally invasive surgical procedure that is regarded as an effective alternative to open micro-discectomy for the treatment of lumbar disc herniation. Many studies published in the literature so far have shown that the clinical outcome of PELD is comparable to those of microsurgical techniques ${ }^{14,18,19)}$. As with any new surgical technique, PELD is associated with various adverse events or complications. Among these complications are dural tears $^{2}{ }^{2}$, nerve root injury ${ }^{6}$, retroperitoneal hematoma ${ }^{1)}$, infections $^{7)}$ and reherniation ${ }^{13)}$. Reherniation after lumbar discectomies (whether open or percutaneous) is considered an inevitable complication. It is defined as recurrence of symptoms secondary to new herniated fragments after a pain-free period of time. 
After surgery, a hidden or loose intradiscal fragment may extrude from the same side or the contralateral side. The rate of reherniation after PELD has been reported in the literature to range from $5 \%$ to over $20 \%{ }^{12,15,19)}$. Risk factors include obesity, older age, degree of disc degeneration, presence of Modic changes, learning curve of the surgeon and central location of herniation ${ }^{4,13,21)}$. Cinotti et al. reported that the remaining nucleus is susceptible to reherniation when mechanically overloaded, and that the rate of recurrence is related to the size of the annular incision during the operation ${ }^{4)}$. According to this study, the patients with a higher BMl showed a higher recurrence rate, since more weight could cause more load on the disc, which results in recurrence. Carragee et al. ${ }^{3)}$ published a prospective study on the recurrence rate after lumbar disc herniation in 187 patients who had micro-discectomy secondary to lumbar disc herniation. He showed that the rate of reherniation is closely related to the defect in the posterior annulus. The creation of an annulotomy during the procedure is likely to increase the risk of reherniation, especially if a large window is made in the annulus for access rather than utilizing a small window. Our patient had a large annular defect as demonstrated by the axial MRI (Fig. 1B) which might have contributed to the early recurrence. This should be taken into consideration when planning surgery for patients with lumbar disc herniation. Patients with large annular defect might be better treated with open lumbar discectomy rather than PELD to decrease the chance of early recurrence.

Different surgical techniques have also been associated with recurrence of disc herniation. Traditionally, subtotal discectomy that entails incision of the annulus with subtotal disc removal including the nucleus down to healthy cartilaginous endplates has been advocated. Later on, sequestrectomy or fragmentectomy has been popularized. This technique entails blunt annular perforation and removal of only those fragments that are easily mobilized. A true fragmentectomy actually does not require opening the annulus, and the disc space is not entered. There are conflicting results in the literature regarding the difference in outcome between subtotal discectomy and fragmentectomy. It was traditionally thought that subtotal discectomy would decrease the risk of recurrence while fragmentectomy is associated with a higher risk of recurrence. Caragee et al. found a rate of $18 \%$ with sequestrectomy compared to $9 \%$ with subtotal discectomy ${ }^{3)}$. Another study found a recurrence rate of $15 \%$ with sequestrectomy and only $6 \%$ in subtotal discectomy ${ }^{20)}$. In contrast, other studies showed that fragmentectomy does not have a higher reherniation rate, but that subtotal discectomy is associated with greater loss of disc height and a higher level of recurrent back pain ${ }^{10,17)}$.

When performing open microdiscectomy, it is possible to remove the disc herniation and adequately examine the intradiscal space for any hidden fragment. However, under endoscopic surgery, the working area is limited and it is difficult to remove hidden fragments which are located beyond the working channel.

Our case is a young, relatively obese, patient who had an
L4-5 preforaminal disc herniation that was treated successfully by transforaminal endoscopic discectomy. The procedure was uncomplicated and the patient was symptom free for 2 days only. She had a massive disc re-herniation at the same level on the same side that resulted in cauda equina syndrome with remarkable weakness in both lower extremities. It is certainly not a hidden fragment or incomplete disc removal as the new disc herniation is much bigger than the original one and it is obliterating the whole spinal canal as it is appears on the second MRI (Fig. 4).

For transforaminal endoscopic discectomy, 2 techniques have been described: the "inside-out" and the "outside-in" technique. In the inside-out technique, the working cannula puncture the annulus first and the tip of the working cannula is located within the disc space. The procedure is continued by enlarging the annular window gradually to localize and remove the herniated disc fragments. For the outside-in technique, the opening of the working cannula does not perforate the annulus and is initially positioned in the foramen or epidural space and then pushed inside the disc space, if needed.

We believe that one possibility for this unusual recurrence in our case is the large annular window left behind secondary to the "inside-out" technique used during the procedure for accessing the disc space.

Many researchers have found that recurrences, like in our case, most frequently occur at L4/5 level and next at L5/S1 level with more acute recurrences found after minimally invasive endoscopic discectomy than after open disc surgery ${ }^{4,12,21)}$. Cheng et al. ${ }^{5)}$ found that, among all reoperations, the percentages of acute reoperations (within 6 months) were higher in the PELD group than in the open group (76.5\% vs. $15.6 \%, p<0.01)$. They claimed that unappreciated disc fragment remnants and incomplete decompression by piecemeal removal may lead to a higher early recurrence. To reduce recurrence rates, they suggested that complete removal of herniated disc must be done including the basal and extruded parts.

\section{CONCLUSION}

Percutaneous Endoscopic lumbar discectomy (PELD) is being performed more frequently for treatment of lumbar disc herniation. we report here a rare case of acute recurrence of disc herniation after a successful PELD done at L4-5 level causing acute cauda equina syndrome. Surgeons performing endoscopic discectomy should be aware of such complication.

\section{REFERENCES}

1. Ahn Y, Kim JU, Lee BH, Lee SH, Park JD, Hong DH, Lee JH: Postoperative retroperitoneal hematoma following transforaminal percutaneous endoscopic lumbar discectomy. J Neurosurg Spine 10(6):595-602, 2009

2. Ahn Y, Lee HY, Lee SH, Lee JH: Dural tears in percutaneous endoscopic lumbar discectomy. Eur Spine J 20(1):58-64, 2011

3. Carragee EJ, Han MY, Suen PW, et al: Clinical outcomes after 
lumbar discectomy for sciatica: the effects of fragment type and anular competence. J Bone Joint Surg Am 85:102-108, 2003

4. Cinotti G, Roysam GS, Eisenstein SM, et al: Ipsilateral recurrent lumbar disc herniation. J Bone Joint Surg [Br] 80:825-832, 1998

5. Cheng J1, Wang H, Zheng W, Li C, Wang J, Zhang Z, Huang B, Zhou Y: Reoperation after lumbar disc surgery in two hundred and seven patients. Int Orthop 37(8):1511, 2013

6. Choi I, Ahn JO, So WS, Lee SJ, Choi IJ, Kim H: Exiting root injury in transforaminal endoscopic discectomy: preoperative image considerations for safety. Eur Spine J 22(11):2481-2487, 2013

7. Choi KB, Lee CD, Lee SH: Pyogenic spondylodiscitis after percutaneous endoscopic lumbar discectomy. J Korean Neurosurg Soc 48(5):455-460, 2010

8. Choi KC, Kim JS, Kang BU, Lee CD, Lee SH: Changes in back pain after percutaneous endoscopic lumbar discectomy and annuloplasty for lumbar disc herniation: a prospective study. Pain Med 12(11):1615-1621, 1526-4637, 2011

9. Dai LY, Zhou Q, Yao WF, Shen L: Recurrent lumbar disc herniation after discectomy: outcome of repeat discectomy. Surg Neurol 64(3):226-231, 2005

10. Fakouri B, Shetty NR, White TC: Is sequestrectomy a viable alternative to microdiscectomy? A systematic review of the literature. Clin Orthop Relat Res 473(6):1957-1962, 2015

11. Kim CH, Chung CK, Park CS, Choi B, Kim MJ, Park BJ: Reoperation rate after surgery for lumbar herniated intervertebral disc disease: nationwide cohort study. Spine (Phila Pa 1976) 38(7):581-590, 2013

12. Kim DH, Choi G, Lee S-H: Complications in percutaneous endoscopic lumbar diskectomy. In: Endoscopic Spine Procedures. Thieme Medical Publishers, Inc., NY, USA, pp253-267, 2011.
13. Kim JM, Lee SH, Ahn Y, Yoon DH, Lee CD, Lim ST: Recurrence after successful percutaneous endoscopic lumbar discectomy. Minim Invasive Neurosurg 50(2):82-85, 2007

14. Lee DY, Shim CS, Ahn Y, Choi YG, Kim HJ, Lee SH: Comparison of percutaneous endoscopic lumbar discectomy and open lumbar microdiscectomy for recurrent disc herniation. J Korean Neurosurg Soc 46(6):515-521, 2009

15. Lee SH, Kang BU, Ahn Y, et al: Operative failure of percutaneous endoscopic lumbar discectomy: a radiologic analysis of 55 cases. Spine 31(10):E285-E290, 2006

16. Mayer HM, Brock M: Percutaneous endoscopic discectomy: surgical technique and preliminary results compared to microsurgical discectomy. J Neurosurg 78(2):216-225, 1993

17. Ran J, Hu Y, Zheng Z, et al: Comparison of discectomy versus sequestrectomy in lumbar disc herniation: a meta-analysis of comparative studies. PLoS One 10(3):e0121816, 2015

18. Ruetten S, Komp M, Merk H, Godolias G: Full-endoscopic interlaminar and transforaminal lumbar discectomy versus conventional microsurgical technique: a prospective, randomized, controlled study. Spine (Phila Pa 1976) 33(9):931-996, 2008

19. Ruetten S, Komp M, Merk H, Godolias G: Recurrent lumbar disc herniation after conventional discectomy. a prospective, randomized study comparing full-endoscopic interlaminar and transforaminal versus microsurgical revision. J Spinal Disord Tech 22: 122-129, 2009

20. Shamji MF, Bains I, Yong E, Sutherland G, Hurlbert RJ: Treatment of herniated lumbar disk by sequestrectomy or conventional diskectomy. World Neurosurgery 82(5):879-883, 2014

21. Suk KS, Lee HM, Moon SH, et al: Recurrent lumbar disc herniation: result of operative management. Spine 26:672-676, 2001 